2. Kxf2 Qxb5 3. Qd6 Qf5+ 4. Kg1 Qb1+, etc.

A further variation from the article: 1. Rd8 Qxb5 2. Qd6 Re8 now makes 3. a4 possible and after 3. ... Qxa4 4. Qe7 Bxf2+ 5. Kxf2, there are no more checks. If after 3. a4 Bxf2+, it amounts to the above variation.

If after 3. a4 Bxf2+ 4. Kg2 Qxa4 (if Bc5, axb5, Bxd6, Rxe8+, Bf8, bxa6) 5. Qe7 Qe4+ 6. Kh3 Qf5+ 7. g4 and the black Bishop shortens the variation as there is no more check on $\mathrm{f} 1$.

This method of winning is well known from chess literature.

It seems to me that chess computers should find the move a 4 without any trouble, it being so obvious. I find it strange that Mr. Botvinnik, eh, I mean CC Sapiens did not find it at all. One has only to remember the famous game Torre-Adams (Njenarokov: Book of Openings - the chapter on the Philidor Defense!).

Further it strikes me that the line mentioned in 'note added in proof' (page 75) allowing the check on b6 is labeled 'a mistake in a subroutine'. To me it is typical of a human oversight during analysis. Mr. Botvinnik should leave the program as it is, because then he can be commended for having written a program that mimics human oversights!

\title{
PUZZLING WITH ICCA
}

\author{
John White \\ 22, Starling Close \\ Woosehill, Wokingham \\ Berks RG11 2YY, England
}

The recent issue of the ICCA Journal (June 1993, Vol. 16, No. 2) provided several puzzling features. In the order of encountering them:

1. M. Botvinnik's account of his CC Sapiens program purports to show that only 18 nodes are required to evaluate fully a complex tactical position for which the key move was d2-d8 (in general, Botvinnik claims success in searching typical chess positions with 'the size of the search tree counting only 10 nodes or even fewer than $10^{\prime}$ ). But how did his program select d2-d8 as the key? Surely some other moves at each ply - and all legal moves at the first ply - were generated and examined at least cursorily, even if only to reject them at once. This adds significantly to the total number of nodes in the search tree.

The significance of this consideration can be seen with I.J. Good's argument later in the same issue of the Journal that the new goal for computer chess should be one in which fewer positions are examined to achieve master play. It is first clearly necessary to decide what a 'position' is. I should like to modify Good's proposal to suggest that the new target should be set to a maximum number of static evaluations (say 10,000 in the first instance, but with the long term objective of aiming for less than 1,000 or even less than 100 static evaluations). For this purpose, a 'static evaluation' may be defined as "an evaluation of a position in which no piece is moved in the computer's internal board representation (or a copy thereof) during the evaluation".

Computer chess does seem to have lost sight of its original objective - to mimic the human thought process.

2. Schaeffer's fascinating account of the checkers' match between Chinook and Dr. Tinsley pointed up a gross mis-match. 
Schaeffer was unhappy with Chinook's comparatively small openings book, but praises its massive end-game database. Both features are the subject of intended improvement. But then Schaeffer remarks (of game 14): "Chinook played an unusual opening line, forcing Dr. Tinsley to recall a 30year old analysis".

How is it that Chinook can refer to electronic databases, but its human adversary cannot resolve his quandary from a printed database (a book)?

Apparently Dr. Tinsley was not permitted by the checkers' governing bodies to play world championship matches against machines (until after he had resigned). On the other hand, the editors of this Journal have demanded, and subsequently applauded, that human chess-players must be forced to play chess computers in serious competitions.

Why so? One might argue by analogy that marathon runners should compete against Formula One racing cars; Olympic swimmers against motor boats. In both cases, the same results are achieved by different processes invoked by human and mechanical competitors, as is currently the case when comparing human and electronic chess play. Yet if the original athletics committees had permitted this, there would have been no human world athletics champions for the last century or so. Is the world chess championship to be held in perpetuity by a series of electronic devices?

3. Published tables of the performance of chess-computer programs are listed in various places in order of their alleged playing strength (as, for example, in the Karlsson/Grottling lists published in each issue of the Journal).

Leaving aside considerations of whether machine-vs-machine contests have any relevance to their play against an inventive human opponent, one cannot help but note that the strongest programs tend to run on the fastest hardware. It would clearly be of considerable interest if a well-known, easily portable Public Domain chess progam - GNU is an obvious candidate - could be entered into such lists on a variety of platforms. Then we would know whether the new, 'super' chess programs are really adding value to that which can easily be achieved on the same platform with old chess-programming technology.

\title{
COMMENT ON 'THE GUARD HEURISTIC'
}

\author{
Jonathan Schaeffer \\ Department of Computing Science \\ University of Alberta \\ Edmonton, Alberta \\ Canada T6G 2H1
}

The previous issue of the ICCA Journal contained an interesting article that proposed a move-ordering mechanism for search trees (Ke and Parng, 1993). The guard heuristic orders moves based on the safety of the move, searching safe moves before unsafe ones. Further, moves that appear to be unsafe are pruned from the search, thereby giving additional savings. In the article, the authors present evidence that their method is a significant improvement over alternative ordering schemes.

The idea of square safety is not new and has been used in several chess programs (for example, Hitech (Ebeling, 1987)). The major contribution of Ke and Parng's (1993) paper is to make the readers more aware of the value of square safety in a move-ordering mechanism. However, their conclusions and some of the claims made about the heuristic's performance are not properly justified. This comment addresses some concerns about the Guard Heuristic paper and the scientific conclusions that can be drawn from it. 